\title{
ZUR THEORIE DER DISCRIMINANTEN
}

voN

\author{
EUGEN NETTO
}

in BERLIN.

Die nachstehende Arbeit knüpft an Untersuchungen an, welche ich früher unter gleichem Titel im 》oJournal für reine und angewandte Mathematik» B. XC, 164-186 veröffentlicht habe. Der Grund, welcher mich bewog, auf diese Untersuchungen zurückzukommen, ist in einigen Bemerkungen, vorzüglich aber in den neuen, grundlegenden Anschauungen zu suchen, die sich in der bedeutenden Abhandlung des Herrn Kronecker: 》Grundzüge einer arithmetischen Theorie der algebraischen Grössen» tinden. $\mathrm{Zu}$ jenen Bèmerkungen zähle ich die (S. 22) gemachte Angabe einer Gleichung, für die es Wurzelgattungen ohne Gattungsdiscriminanten giebt; sie rief die Untersuchungen in $\S 5$ und $\S 6$ der nachfolgenden Arbeit hervor; - ferner den Satz auf S. 42 ( $\$ 13)$ über Functionen einer Gattung, deren conjugịte sämmtlich von einander verschieden sind; sie veranlasste die Ableitungen in $\S 2$.

Besonders aber waren es die im zweiten 'Teile der Kronecker'schen Abhandlung auseinandergesetzten Principien aber Divisorensysteme, welche mich bewogen, den einfachen Fall der Discriminanten algebraischer Gleichungen in dem neuen, überraschenden lichte dieser tiefliegenden und doch ihrem Wesen nach so einfachen Grundanschauungen von neuem zu behandeln. Die algebraische und die substitutionentheoretische Methode der Untersuchung weisen sich hierbei, als in wichtigen Punkten einander erganzend aus, so dass es nicht angethan schien, die eine zn Gunsten der anderen zu unterdrücken. 


\section{$\S 1$.}

Es ist für unsere Untersuchungen ein Punkt von besonderer Wichtigkeit, dass auf die Natur der Discriminante

$$
D_{\varphi}=\left(\varphi_{1}-\varphi_{2}\right)^{2}\left(\varphi_{1}-\varphi_{3}\right)^{2} \cdots\left(\varphi_{\rho-1}-\varphi_{p}\right)^{2}
$$

einer gegebenen $\rho$-wertigen Function $\varphi\left(x_{1}, x_{2}, \ldots x_{n}\right)$ der $n$ von einander unabhängigen Grössen $x_{1}, x_{2}, \ldots x_{n}$ nur solche algebraischen Bezichungen von wesentlichem Einfluss sind, welche in der Gleichsetzung zweier oder mehrerer der Elemente $x$ bestehen.

Un den Kern dieser Verhaltnisse klarzulegen, müssen wir einige elementare und fundamentale Theoreme vorausschicken, deren Bedeutung darin liegt, dass zwischen vorher willkürlichen Grössen bestirnmte algebraische Relationen eingefuhrt werden; dass also sogenannte mallgemeine» Satze bei tatsächlich gegebenen Verhaltnissen Anwendung finden sollen.

Wir verstehen unter $\alpha_{0}, \alpha_{1}, \alpha_{2}, \ldots \alpha_{n}$ willkürliche Constanten, unter $x_{1}, x_{2}, \ldots, x_{n}$ vorläufig unbestimmte Grössen und betrachten die lineare Verbindung der $x$

$$
\psi_{1}=\alpha_{0}+\alpha_{1} x_{1}+\alpha_{2} x_{2}+\cdots+\alpha_{n} x_{n} .
$$

Bei allen möglichen Vertauschungen der $x$ untereinander erhält $\phi_{1}$ eine Reihe von Werten, die ihre Formen nach von einander verschieden sind. Die Operationen der Vertauschungen bezeichnen wir als Substitutionen; es giebt deren $n$ !, falls man diejenige Substitution mitzahlt, welche keins der $x$ von seiner Stelle rückt. Es mögen $s_{1}, s_{2}, \ldots s_{n}$ : diese Substitutionen sein, und $s_{1}$ soll die let\%t erwahnte identische Substitution bedeuten. Wir verstehen ferner unter $\psi_{a}$ denjenigen Wert von $\psi_{1}$, der durch die Anwendung von $s_{a}$ aus $\psi_{\mathrm{s}}$ hervorgeht. (Setzen wir etwa

$$
\chi_{1}=\beta_{0}+\beta_{1} x_{1}+\beta_{2} x_{2}+\cdots+\beta_{n} x_{n},
$$

so sei auch hier $\chi_{a}$ der Wert von $\chi$ welcher durch $s_{u}$ aus $\chi_{1}$ hervorgerufen wird.) Der Index des Ausdruckes wird also durch die Substitution bestimmt.

Falls man für die $x$ besondere Werte einsetzt, brauchen die $n$ ! Ausdrücke $\psi_{u}$ ihren Werten nach nicht sämmtlich von einander verschieden 
zu sein. Sind einige der $x$ untereinander gleich, so ist eine derartige Verschiedenheit nicht einmal mehr möglich. Über die hierbei eintreten. den Umstände war bisher, meines Wissens, nur der folgende Sat $\%$ bekannt: Es können beliebige Beziehungen algebraischer Art zuischen $x_{1}, x_{2}, \ldots . x_{n}$ bestehen; so bald durch dieselben nur nicht die Gleichheit einiger der Elemente $x$ festgesetzt wivd, ist es stets möglich, beliebig viele Systeme $a_{0}, \alpha_{1}, \ldots \alpha_{n}$ der Constanten $\alpha$ zu liefern, fiir welche alle $n$ ! Ausdrïcke $\phi_{1}, \phi_{2}, \ldots \phi_{n}$ ! ihren numerischen Werten nach von einander verschieden sind. (1)

Diesen Satz wollen wir erweitern und die neue Fassung beweisen. Die Erweiterung bezieht sich auf Folgendes: Wenn für Gleichsetzungen der $x$ gewisse Gruppen von Werten der $\psi$ bei jeder Wahl der $\alpha$ einander gleich werden, dann kann der hierdurch bestimmte Charakter dadurch nicht geändert werden, dass man neue Beziehungen unter den $x_{1}, \ldots x_{n}$ festlegt, welche keine neuen Gleichsetzungen der $x$ zur Folge haben.

Wir setzen voraus, es wäre etwa

$$
x_{a_{1}}=x_{a_{2}}=\cdots=x_{a_{\mu}} ; \quad x_{b_{1}}=x_{b_{2}}=\cdots=x_{b_{\nu}} .
$$

Dann werden ersichtlich von den $n !$ Ausdrücken $\phi_{1}, \ldots \phi_{n !}$ genau $\frac{n !}{\mu ! \nu !}=\sigma$ Gruppen von je $\mu$ : $!$ Ausdrücken je einen und denselben Wert annehmen, so dass für $\phi$ höchstens $\sigma$ verschiedene Werte bestehen können. Einem beliebigen $\phi_{\alpha}$ werden nämlich alle diejenigen $\phi$ gleich, welche aus ihm durch Substitutionen hervorgehen, in denen nur $x_{a_{1}}, x_{a_{2}}, \ldots x_{a_{\mu}}$ und ebenso $x_{b_{1}}, x_{b_{3}}, \ldots x_{b_{y}}$ unter sich vertauscht werden. Wir wollen diese Substitutionen, deren Anzahl $\mu$ !L! ist, zum Unterschiede von den übrigen mit $t_{1}, t_{2}, t_{3}, \ldots$ bezeichnen; wir behalten aus jeder der $\sigma$ Gruppen einen Repräsentanten zurück, $\phi_{1}^{\prime},{\psi_{2}^{\prime}}_{2}, \ldots \psi_{\sigma}^{\prime}$. Jetzt nehmen wir weiter an, es träten neue Beziehungen unter den $x$ auf, welche durch die irreductiblen Gleichungen

$$
F_{1}\left(x_{1}, \cdots x_{n}\right)=0, \cdots, F_{k}\left(x_{1}, \cdots x_{n}\right)=0
$$

dargestellt werden, und über deren Natur lediglich das Eine festgesetzt sei, dass durch sie keine weiteren Gleichsetzungen unter den $x$ hervorgerufen werden. Dann lassen sich, wie die $F_{k}$ auch sonst beschaffen sind,

(') Vgl. meine 》Substitutionentheorie» $\$ 32$. 
unendlich viele Systeme der $\alpha_{0}, \alpha_{1}, \ldots \alpha_{n}$ derart bestimmen, dass nach wie vor $\psi_{1}^{\prime}, \phi_{2}^{\prime}, \ldots \psi_{\sigma}^{\prime}$ von einander verschieden bleiben, dass es demnach $\sigma$ numerisch von einander verschiedene Werte des $\psi$ giebt.

Um den Beweis zu führen, setzen wir

$$
\phi_{k}^{\prime}-\phi_{\lambda}^{\prime}=a_{1}\left(x_{k_{1}}-x_{\lambda_{1}}\right)+\alpha_{2}\left(x_{k_{2}}-x_{\lambda_{2}}\right)+\cdots+\alpha_{n}\left(x_{k_{n}}-x_{\lambda_{n}}\right) .
$$

Hier werden $\mu$ der $x_{k}$ und ebensoviele der $x_{\lambda}$ gleich $x_{a_{1}} ; \nu$ der $x_{k}$ und ebensoviele der $x_{\lambda}$ gleich $x_{b_{1}}$. Unseren Festsetzungen gemasss können die Coefficienten der a nicht sämmtlich verschwinden; denn dies trăte nur dann ein, wenn $\psi_{\lambda}^{\prime}$ aus $\psi_{k}^{\prime}$ durch eine Substitution $t$ ableitbar wäre, was ja aber ausgeschlossen ist. Wir betrachten das uber alle Combinationen von je zwei $\psi^{\prime}$ ausgedehnte Product

$$
\Pi\left(\psi_{k}^{\prime}-\psi_{\lambda}^{\prime}\right)=\Pi\left[\alpha_{1}\left(x_{k_{1}}-x_{\lambda_{1}}\right)+\alpha_{2}\left(x_{k_{2}}-x_{\lambda_{2}}\right)+\cdots+\alpha_{n}\left(x_{k_{n}}-x_{\lambda_{n}}\right)\right] .
$$

Unsere Behauptung kann jetzt die Form annehmen: das aufgestellte Product verschwindet nicht för alle Wertsysteme der $\alpha$. Wir sortiren die Factoren des Products der rechten Seite; zuerst betrachten wir die, bei denen der Coefficient von $\alpha_{1}$ nicht Null ist; dann die, bei denen er Null ist, wahrend der von $\alpha_{2}$ nicht verschwindet; dann die, bei denen die beiden ersten Coefficienten Null sind, der dritte jedoch nicht; u. s. f. Bei der ersten. Klasse können wir es durch passende Wahl von $\alpha_{1}$, ohne Beschränkung von $a_{2}, a_{3}, \ldots$ dahin bringen, dass die zugehörigen Factoren nicht Null werden. Dazu ist nur nőtig, dass $\alpha_{1}$ keinen der Werte

$$
-\frac{\alpha_{3}\left(x_{k_{2}}-x_{\lambda_{2}}\right)+\cdots+\alpha_{n}\left(x_{k_{n}}-x_{\lambda_{n}}\right)}{x_{k_{1}}-x_{\lambda_{1}}}
$$

erhalt, deren Zahl endlich ist und deren Wert wegen des nicht verschwindenden Nenners angebbar sein wird. Bei der zweiten Klasse kőnnen wir dasselbe durch passende Wahl von $\alpha_{2}$ ohne Beschränkung von $\alpha_{3}, \alpha_{4} \ldots$. erlangen u. s. f. Wahlt man demgemass $\alpha_{n}$ in den Factoren der letzten Klasse (für welche die Coefficienten von $a_{1}, a_{2}, \ldots \alpha_{n-1}$ verschwinden) ganz beliebig, bestimmt $\alpha_{n-1}$ durch das Nichtverschwinden der Factoren der vorletzten Klasse $u$. s. f., dann erhalt man Systeme von $\alpha_{1}, \alpha_{2}, \ldots \alpha_{n}$, für die kein $\psi_{k}^{\prime}$ einen $\psi_{2}^{\prime}$ gleich wird. Eine Einwirkung der $F_{1}, F_{2}, \ldots F_{k}$ 
ist also überhaupt nicht zu bemerken. Hieraus folgt: Auf lie Gleichheit der verschiedenen Werte von linearen Ausdrücken der Form

$$
\psi=\alpha_{0}+\alpha_{1} x_{1}+\alpha_{2} x_{2}+\cdots+\alpha_{n} x_{n}
$$

haben nur diejenigen Beziehungen unter den $x$ bestimnenden, d. h. von der Wahl der a unabhängig bestehenden Einfluss, welche in der Gileichsetzung einzelner $x$ selbst begründet sind.

\section{$\S 2$.}

Wir setzen zunächst für den ersten Teil dieses Paragrafen die $x$ wieder als völlig unbestimmte Grössen voraus.

Einer jeden unter den $x_{1}, x_{2}, \ldots x_{n}$ inöglichen Functionengattung $\left({ }^{1}\right)$ entspricht eine charakteristische Substitutionengruppe, welche odie Permutationen der Gattung» enthalt. Dies heisst: die Gruppe $G$ der Gattung (f) umfasst alle und auch nur diejenigen Substitutionen, welche die Form der sämmtlichen zur Gattung $\mathbb{B S}$ gehörigen Functionen $y, y^{\prime}, y^{\prime \prime}, \ldots$ nicht verändern. Wenn diese Substitutionen

$$
s_{1}=1, s_{2}, s_{3}, \cdots s_{r}
$$

sind, so deuten wir die Gruppe $G$ wohl auch kurz durch

$$
G=\left[s_{1}, s_{2}, s_{3}, \cdots s_{r}\right]
$$

an. Die Zahl $r$, die Ordnung der Gruppe, zeigt demnach, für wie viele Substitutionen die Functionen $y, y^{\prime}, y^{\prime \prime}, \ldots$ der Gattung (5) ungeändert bleiben; hieraus folgt, dass diese Functionen je $\frac{n !}{r}=\rho$ verschiedene Werte besitzen. Die zu $y^{(\alpha)}$ gehörigen mögen $y_{1}^{(a)}, y_{2}^{(\alpha)}, \ldots y_{\rho}^{(\alpha)}$ sein, derart, dass wir $y^{(a)}$ auch $=y_{1}^{(\alpha)}$ setzen; alle diese Werte heissen die sconjugirten Werte) von $y^{(\alpha)}$; sie bestimmen sconjugirte Gattungen) $\mathscr{B}_{1}, \mathscr{G}_{2}, \ldots \mathscr{G}_{\rho}$, wo wir auch $\mathbb{B}$ gleich $\mathscr{S}_{1}$ annehmen; zu ihnen gehören sconjugirte Gruppen» $G_{1}, G_{2}, \ldots G_{\rho}$, wo endlich auch $G$ mit $G_{1}$ identisch sein soll. Die $\rho$ conjugirten Werte

( $\left.{ }^{1}\right)$ Über die hier benutzte Terminologie, welche sich der von Herrn Kronecker gebrauchten genau anschliesst vgl. L. KRONECKER: Grundzüge einer arithmetischen Theorie der algebraischen Grössen; S. $6 \mathrm{ff}$. 
einer Function $y$ sind die Wurzeln einer Gleichung $\rho^{\text {ten }}$ Grades, deren Coefficienten dem aus den elementaren symmetrischen Functionen

(1) $\mathfrak{f}_{1}=x_{1}+x_{2}+\cdots+x_{n}, \quad \mathfrak{f}_{2}=x_{1} x_{2}+x_{1} x_{3}+\cdots+x_{n-1} x_{n}, \cdots \quad \mathfrak{f}_{n}=x_{1} x_{2} \cdots x_{n}$

gebildeten Rationalitätsbereiche angehören.

Da conjugirte Werte aus einander durch Anwendung gewisser Substitutionen hervorgehen, so besitzen sie sämmtlich denselben Typus. Wir wollen eine der Substitutionen, welche $y_{1}$ in $y_{k}$ umwandelt mit $\sigma_{k}$ bezeichnen; dann erhält man sämmtliche Substitutionen derselben Eigenschaft durch $s_{\lambda} \sigma_{k}(\lambda=1,2, \ldots r)$ oder kurz durch $G_{1} . \sigma_{k}$. Ist $y^{(a)}$. eine andere Function derselben Gattung, so bezeichnen wir mit $y_{k}^{(\alpha)}$ denjenigen ihrer conjugirten Werte, welcher aus $y_{1}^{(\alpha)}$ durch $G_{1} \cdot \sigma_{k}$ abgeleitet werden kann.

Sämmtliche $n$ ! Substitutionen lassen sich in die folgende Tabelle einordnen

$$
\begin{aligned}
& s_{1}=1, \quad s_{1}, \cdots s_{r} ; \quad \mathbb{S}_{1} \\
& s_{1} \sigma_{3}, \quad s_{2} \sigma_{2}, \ldots s_{r} \sigma_{3} ; \quad \mathscr{G}_{3} \\
& \ldots \ldots \ldots \ldots \ldots \\
& s_{1} \sigma_{\rho}, \quad s_{2} \sigma_{\rho}, \cdots s_{r} \sigma_{\rho} ; \quad \mathscr{G}_{\rho}
\end{aligned}
$$

bei welcher die $i^{\text {te }}$ Zeile alle diejenigen und nur die Substitutionon enthält, welche $y_{1}^{(a)}$ in $y_{i}^{(a)}$ umwandeln.

Wir gehen nach der Recapitulation dieser bekannten Begriffe und Bezeichnungen dazu über, Functionen $y^{(\alpha)}$ mit Hülfe der im ersten Paragrafen aufgestellten $\phi$ zu bilden.

Wenden wir $G_{1}$ auf $\phi_{1}$ an, so entstehen dadurch $r$ Ausdrücke

$$
\phi_{1}, \psi_{2}, \phi_{3}, \cdots, \phi_{r}
$$

welche ihrer Form nach von einander verschieden sind. Jede symmetrische Function derselben bleibt für $G_{1}$ ungeändert und gehört also zur Gattung $\mathscr{B}_{1}$ oder $\mathrm{zu}$ einer unter $\mathscr{S}_{1}$ enthaltenen Gattung. Richtet man jedoch die symmetrische Function so ein, dass sie nur dann ungeăndert bleibt, wenn die $\psi$ mit einander vertauscht werden, dann gehört sie wirklich zur Gattung $\mathscr{G}_{1}$. Bei

$$
y=\psi_{1}^{e}+\psi_{2}^{e}+\psi_{3}^{e}+\cdots+\psi_{r}^{e}
$$


kann der erstere Fall für $e=1$ cintreten; denn hier bleibt inöglicherweise $\psi_{1}$ auch ungeändert für Substitutionen, welche nicht $z u G_{1}$ gehören. Für ein unbestimmtes $e$ geht dies aber nicht mehr an.

Aus der Tabelle (C) erkennt man, dass alle $n$ ! Ausdrücke von $\phi$ in die $\rho$ conjugirten $y_{1}, y_{2}, \ldots y_{\rho}$ als Summanden eingehen; jeder Wert von $\psi^{\prime}$ kommt überhaupt nur einmal vor; es ist also schon durch das Auftreten eines $\phi_{n}$ das zugehörige $y_{o}$ unzweideutig bestimnt: es ist dasjenige, in dessen Zeile der Tabelle (C) die Substitution $s_{n}$ auftritt, welche $\phi_{1}$ in $\psi_{n}$ umändert. Dieselbe Substitution $s_{n}$ führt demnach auch $y_{1}$ in $y_{o}$ über. Allgemeiner: enthält $y_{k}$ den Summanden $\phi_{k}^{e}$, $y_{2}$ den Summanden $\phi_{i}^{e}$ und geht $\phi_{k}$ in $\psi_{2}$ durch die Substitution - über, so geht auch $y_{k}$ in $y_{2}$ durch dieselbe Substitution über.

Wir haben bisher in diesern Paragrafen die $x$ als unbestinmte Grössen angenommen; von jetzt ab wollen wir die Voraussetzungen (A) und (B) des ersten Paragrafen gelten lassen. Wir wăhlen die $\alpha$ derart, dass

$$
\psi_{1}^{\prime}, \psi_{2}^{\prime}, \cdots \psi_{\sigma}^{\prime} \quad\left(\sigma=\frac{n !}{\mu ! \nu !}\right)
$$

von einander verschieden sind. Dann erkennt man ohne jede Schwierigkeit, dass die Willkür, die bei der Wahl von $\alpha_{0}$ herrscht, dazu benutzt werden kann, auch die Moduln der $\phi^{\prime}$ von einander verschieden zu machen. Hierbei sei

$$
\bmod . \psi_{1}^{\prime}>\bmod \psi_{2}^{\prime}>\cdots>\bmod . \psi_{\sigma}^{\prime} \text {. }
$$

Fndlich kann man den ganzzahligen, sonst aber beliebigen Exponenten $e$ so gross annehmen, dass man erbält

$$
\begin{aligned}
& \phi_{1}^{\prime \prime e}>\rho\left(\phi_{2}^{\prime e}+\phi_{3}^{\prime e}+\cdots+\phi_{\sigma}^{\prime \prime}\right) \\
& \psi_{i}^{\prime \prime}>\rho\left(\psi_{3}^{\prime e}+\psi_{4}^{\prime \prime e}+\cdots+\psi^{\prime \prime}\right) \\
& \phi_{\sigma-1}^{\prime \prime}>\rho \cdot \phi_{\sigma}^{\prime e}
\end{aligned}
$$

der Kürze halber sind die $\phi^{\prime}$ statt ihrer Moduln hingeschrieben. Aus unseren Festsetzungen ergiebt sich, dass eine Gleichung

$$
\begin{aligned}
& m_{1} \phi_{1}^{\prime \prime}+m_{2} \psi_{2}^{\prime e}+\cdots+m_{\sigma} \psi_{\sigma}^{\prime e}=\mu_{1} \psi_{1}^{\prime e}+\mu_{2} \psi_{2}^{\prime e}+\cdots+\mu_{\sigma} \psi_{\sigma}^{\prime \prime e} \quad(m, \mu \leqq \rho) \\
& \text { Actu mathematira. I. }
\end{aligned}
$$


nur dann bestehen kann, wenn alle entsprechenden $m, \mu$ einander gleich sind.

Unter den jetzt gelienden Annahmen (A), (B) geht die oben gebildete Function $y_{i}$ aus ihrer allgemeinen Gestalt

$$
y_{i}=\phi_{i_{1}}^{e}+\phi_{i_{2}}^{e}+\cdots+\xi_{i_{r}}^{e}
$$

in die besondere Form

$$
\dot{y_{i}}=m_{i_{1}} \psi_{i_{1}}^{\prime e}+m_{i_{2}} \psi_{i_{2}}^{\prime e}+\cdots+m_{i_{t}} \psi_{i_{i}}^{\prime e}
$$

über, da gewisse Werte $\phi_{i_{1}}, \phi_{i_{2}}, \ldots$ gleich $\psi_{i_{1}}^{\prime}$ u. s. w. werden können. Sollte nun durch (A), (B) eine Gleichheit zweier conjugirter Werte hervorgerufen werden, etwa

$$
y_{i}=y_{k},
$$

so folgt wegen (3) dass $y_{i}, y_{k}$ dieselben Summanden $\psi_{\alpha}^{\prime e}$ enthalter. Dies erlaubt den Ruckschluss, dass vor dem Gelten von (A), (B) beide conjugirte Werte $y_{i}, y_{k}$ nur Summanden derjenigen Gruppe enthielten, als deren Repräsentanten wir $\phi^{\prime \prime}{ }_{\alpha}$ zurückbehalten haben. Zwei derartige Summanden konnen durch Substitutionen $t$ in cinander verwandelt werden, in denen nur die $x_{a_{1}}, x_{a_{2}}, \ldots x_{a_{a_{4}}}$ und die $x_{b_{1}}, x_{b_{2}}, \ldots x_{b_{y}}$ sich untereinander umstellen. Nach unseren obigen Ausführungen leitet dann $t$ den ganzen Summandencomplex der Function $y_{i}$ in den von $y_{k}$ über.

Dass umgekehrt alle diejenigen Functionen, welche durch Substitutionen $t$ aus einander hervorgehen, durch (A), (B) einander gleich werden, ist selbstverstandlich; es geht aber aus unserer Beweisfährung auch hervor, dass dieser a priori ersichtliche Fall auch der einzige ist, in welchem conjugirte Werte $y_{i}^{(\alpha)}, y_{k}^{(\alpha)}$ einer jeden Function $y^{(\alpha)}$ der Gattung einander gleich werden; und es ist von Wichtigkeit zu bemerken, dass nur die Bedingungen (A) nicht aber die Festsetzungen (B) einen Finfluss dabei haben.

Herr KRONECK $\mathrm{ER}$ hat gezeigt, $\left({ }^{1}\right)$ )dass sich für irgend welche gegebenen Werte $\mathfrak{f}_{\mathfrak{j}}, \mathfrak{f}_{2}, \ldots, \mathfrak{f}_{n}$ von $(1)$ - vorausgesetzt nur, dass nicht zwei der $x$ einander gleich werden - stets unendlich viele specielle Functionen jeder Gattung \&S bestimmen lassen, deren sïmmtliche conjugirte unter einander ver-

(') Grundzüge einer arithmetischen Theorie a. s. w., S. 42 . 
schieden sind.) Diesem Satze können wir jetzt folgende Erweiterung zur Seite stellen: Auf die Gleichheit conjugirter Werte aller Functionen einer Gattung haben nur diejenigen Beziehungen unter den $x$ einen bestimmenden Einfluss, welche in der Gleichsetzung einzelner $x$ bestehen.

Beachtenswert ist ferner noch der Umstand, dass wir Functionen $y$ construirt haben, bei denen aus $y_{i}=y_{k}$ auf die Existenz der Substitution $t$ geschlossen werden kann, welche $y_{i}$ in $y_{k}$ verwandelt. Denn weil die Substitution von der Wahl der Function $y$ unabhängig ist, so folgt, dass für jedes beliebige $y^{(\alpha)}$ stets $y_{i}^{(\alpha)}=y_{k}^{(\alpha)}$ sein wird. Das Gleichwerden conjugirter Werte findet also seinen genauen Ausdruck in Gruppeneigenschaften. Somit ist auch ersichtlich: Verschwindet die Discriminante $D_{y}$ jeder beliebigen Function $y$ der Gattung (B) für gewisse Beziehungen $(A),(B)$ unter den $x$, so ist dieses Verschwinden nicht nur für $I_{y}$, sondern auch für gewisse Differenzen $y_{i}-y_{k}$ invariant.

\section{$\$ 3$.}

Der zuletzt aufgestellte Satz erlaubt uns, statt des Verschwindens der Discriminante selbst, dasjenige der Differenzen $y_{i}-y_{k}$ zu untersuchen.

Herr KRONECKER hat für Functionen mit mehreren Veränderlichen eine Erweiterung des Begriffes der Division gegeben. $\left({ }^{1}\right)$ Verschwindet eine ganze Function $g\left(x_{1}, x_{2}, \ldots x_{n}\right)$ der Veränderlichen $x_{1}, x_{2}, \ldots x_{n}$ sobald gewisse Beziehungen platzgreifen, welche durch die Gleichungen

$$
F_{1}\left(x_{1}, \cdots x_{n}\right)=0, \cdots, F_{k}\left(x_{1}, \cdots x_{n}\right)=0
$$

dargestellt werden, dann ist $g$ als ganze lineare Function von $F_{1}, \ldots, F_{k}$ darstellbar, mit Coefficienten, welche rationale ganze Function von $x_{1}$, $x_{2}, \ldots, x_{n}$ sind:

$$
g\left(x_{1}, \cdots x_{n}\right)=P_{1}\left(x_{1}, \cdots x_{n}\right) F_{1}\left(x_{1}, \cdots x_{n}\right)+\cdots+P_{k}\left(x_{1}, \cdots x_{n}\right) P_{k}\left(x_{1}, \cdots x_{n}\right) .
$$

Wir schreiben dies den Einführungen des Herrn Kronecker entsprechend

$$
\left.g\left(x_{1}, \cdots x_{n}\right) \equiv 0, \quad \text { (modd. } F_{1}, F_{\grave{2}}, \cdots, F_{k}\right)
$$

nennen $F_{1}, F_{2}, \ldots, F_{k}$ ein »Modul-oder Divisoren-System $k^{\text {ter }}$ Stufen von $g$, und sagen, dass $g$ dieses Divisorensystem enthält.

(') a. a. 0.8 .72 f. 
Hiernach können wir die Resultate unserer bisherigen Untersuchungen folgendermassen aussprechen:

1) Jedes Modulsystem der Discriminanten $D_{y}$ der Gattung (S) ist gleichzeitig Modulsystem einer bestimmten und durch die Gruppe $G$ der Gattung bestimmbaren Differenz $y_{i}-y_{k}$, unabhängig von der Wahl von $y$.

2) Alle Modulsysteme der Discriminanten $D_{y}$ bez. der Differenzen $y_{i}-y_{k}$ bestehen aus Functionen der Form

$$
F_{a, \beta}^{\prime}=x_{\alpha}-x_{\beta} \text {. }
$$

3) Die Modulsysteme von $y_{i}-y_{k}$ werden sümmtlich durch diejenigen Substitutionen bestimmt, welche $y_{i}$ in $y_{k}$ umwandeln. Hierzu ist es nur nötig, dass alle diejenigen Elemente $x$, welche je einen Cyklus einer solchen Substitution bilden, einander gleich gesetzt werden.

Es fragt sich nun umgekehrt, ob aus jeder Substitution, welche $y_{i}$ in $y_{k}$ umwandelt, ein Modulsystem gebildet werden kann. Dies ist nach dem, am Schlusse des vorigen Paragrafen Besprochenen zwar an und für sich klar; jedoch müssen einige hierbei auftretende Punkte noch weiter untersucht werden. Dieselben beziehen sich auf die Zusammensetzung von Modulsystemen. Wir wollen zunăchst an einem einfachen Beispiele die Frage klarstellen.

Es sei in dem Bereiche der vier Variablen $x_{1}, x_{2}, x_{3}, x_{4}$ eine Functionengattung $\mathbb{S}$ durch

$$
G_{1}=\left[1,\left(x_{1} x_{2} x_{3}\right),\left(x_{1} x_{3} x_{2}\right)\right]
$$

bestimmt. Eine der zur Gattung gehörigen Functionen ist

$$
y_{1}=x_{1}^{\alpha} x_{2}^{\beta}+x_{2}^{\alpha} x_{3}^{\beta}+x_{z}^{\alpha} x_{1}^{\beta} .
$$

Der conjugirte Wert

$$
y_{2}=x_{4}^{\alpha} x_{2}^{\beta}+x_{2}^{\alpha} x_{3}^{\beta}+x_{3}^{\alpha} x_{4}^{\beta}
$$

von $y_{1}$ ist durch die Substitutionen

$$
G_{1} s_{2}=\left\{\left(x_{1} x_{4}\right),\left(x_{1} x_{2} x_{3} x_{4}\right),\left(x_{1} x_{3} x_{2} x_{4}\right)\right\}
$$

aus dem ersteren Werte entstanden. Wir erhalten demnach drei in $y_{1}-y_{2}$ enthaltene Divisorensysteme, bemerken aber zugleich, dass verschiedene Substitutionen gleiche Modulsysteme hervorrufen können, sowie dass einige 
Modulsystcme erfüllt sein können, falls bereits andere cinfachere befriedigt sind. Die hier auftretenden sind

$$
\text { I) } x_{1}-x_{4} ; \quad \text { II) } x_{1}-x_{4} ; x_{1}-x_{2} ; x_{1}-x_{3} \text {; }
$$

Dass jedes derselben wirklich in

$$
\begin{aligned}
y_{1}-y_{2} & =\left(x_{1}^{\alpha}-x_{4}^{\alpha}\right) x_{2}^{\beta}+\left(x_{1}^{\beta}-x_{4}^{\beta}\right) x_{3}^{\alpha} \\
& =\left(x_{1}-x_{4}\right)\left\{\left[x_{1}^{\alpha-1}+x_{1}^{\alpha-2} x_{4}+\cdots\right] x_{2}^{\beta}+\left[x_{1}^{\beta-1}+x_{1}^{\beta-2} x_{4}+\cdots\right] x_{3}^{a}\right\}
\end{aligned}
$$

enthalten ist, leuchtet ein; dass ferner ein aus I), II) componirtes System $\left(^{1}\right)$ nicht vorkornmt, ist gleichfalls daraus ersichtlich, dass der zweite Factor überhaupt für keine Gleichsetzungen der $x$ mehr zu Null wird.

Wir werden daher, um die Divisoren von $y_{k}-y_{i} z$ ermitteln, aus den Substitutionen des Complexes $G_{i} s_{k}$ nur diejenigen zur Bildung von Modulsystemen verwenden, bei denen die Elemente der einzelnen Cykel nicht gänzlich in den Elementen der einzelnen Cykel anderer Substitutionen auftreten. (Die früher von mir benutzte Benennung des „Enthaltenseins), durch welche ich diese Bezjehung von Substitutionen zu einander andeutete, gebe ich auf, da sie mit der bei Modulsystemen gebräuchlichen Verwendung dieses Wortes nicht abereinstimmt.)

Ein zweites Bespiel mag auf eine weitere hier auftretende Eigentümlichkeit aufmerksam machen. Es sei, wieder in dem Bereiche von vier Variabeln

dann wird

$$
\begin{aligned}
& G_{1}=\left[1,\left(x_{1} x_{2} x_{3} x_{4}\right),\left(x_{1} x_{3}\right)\left(x_{2} x_{4}\right),\left(x_{1} x_{4} x_{3} x_{2}\right)\right] \\
& y_{1}=x_{1} x_{2}{ }^{2}+x_{2} x_{3}{ }^{2}+x_{3} x_{4}{ }^{2}+x x_{1}{ }^{2} \\
& y_{2}=x_{1} x_{4}{ }^{2}+x_{4} x_{3}{ }^{2}+x_{3} x_{2}{ }^{2}+x_{2} x_{1}{ }^{2}
\end{aligned}
$$

$$
\begin{aligned}
G_{1}^{Y} s_{2} & =\left[\left(x_{1} x_{3}\right),\left(x_{1} x_{2}\right)\left(x_{3} x_{4}\right),\left(x_{2} x_{4}\right),\left(x_{1} x_{4}\right)\left(x_{2} x_{3}\right)\right] \\
y_{1}-y_{2} & =\left(x_{1}-x_{3}\right)\left(x_{2}-x_{4}\right)\left[x_{2}+x_{4}-x_{1}-x_{3}\right] .
\end{aligned}
$$

Das Auftreten von $\left(x_{1} x_{3}\right)$ und von $\left(x_{2} x_{4}\right)$ in dem Complexe $G_{1} s_{2}$ äussert sich durch das Vorhandensein der beiden Factoren $\left(x_{1}-x_{3}\right)$ und

( ${ }^{1}$ Vgl. KronkCkER, a. a. O. S. 77 ff. - sowie auch die weiteren Ausführungen dieses Paragrafen. 
$\left(x_{2}-x_{1}\right)$; dasjenige von $\left(x_{1} x_{2}\right)\left(x_{3} x_{4}\right),\left(x_{1} x_{4}\right)\left(x_{2} x_{3}\right)$ durch das Verschwinden des letzten Factors

$$
\psi=\left(x_{2}+x_{1}\right)-\left(x_{1}+x_{3}\right)
$$

für die beiden Gleichungssysteme

$$
\text { I) } x_{1}-x_{2}=0, x_{3}-x_{4}=0 ;, \quad \text { II) } x_{1}-x_{4}=0, x_{2}-x_{3}=0,
$$

welche den Modulsystemen

$$
\text { I') } x_{1}-x_{2}, x_{3}-x_{4} ; \quad \text { II') } x_{1}-x_{4}, x_{2}-x_{3}
$$

entsprechen. Dass $\psi$ aber auch das aus $\left.\mathrm{I}^{\prime}\right), \mathrm{II}^{\prime}$ ) componirte Modulsystem in sich schliesst, ist nicht unmittelbar ersichtlich noch auch richtig.

Wir beschäftigen uns zunăchst mit der Klarung dieser Verhaltnisse;

- dazu massen wir, wie dies ja bei dem vorliegenden Thema natürlich ist, auf die grundlegenden Untersuchungen des Herrn Kronecker unser Augenmerk richten. Wir entnehmen denselben folgende begrifflichen Festsetzungen: $\left({ }^{1}\right)$

I. Es ist für zwei Functionen $M, M^{\prime}$ von $n$ Veränderlichen $x_{1}, x_{2}, \ldots, x_{n}$

$$
M \equiv M \quad\left(\operatorname{modd} . M_{1}, M_{2}, M_{3}, \cdots\right),
$$

wenn die Differenz $M-M^{\prime}$ das Modulsystem $\left(M_{1}, M_{2}, M_{3}, \ldots\right)$ enthält.

II. Ein Modulsystem $\left(M_{1}, M_{2}, \ldots\right)$ enthalt ein anderes $\left(M_{1}^{\prime}, M_{2}^{\prime}, \ldots\right)$ wenn jedes Element des ersteren das Modulsystem $\left(M_{1}^{\prime}, M_{2}^{\prime}, \ldots\right)$ enthalt. Wenn jedes der beiden Modulsysteme das andere enthălt, so sind sie einander aequivalent, und dies wird durch: $\left(M_{1}, M_{2}, \ldots\right) \sim\left(M_{1}^{\prime}, M_{2}^{\prime}, \ldots\right)$ bezeichnet.

III. Ein Modulsystem $\left(M_{1}, M_{2}, \ldots\right)$, dessen einzelnen Elemente durch die verschiedenen Producte von je zwei Elementen $M_{k}{ }_{k} M^{\prime \prime}{ }_{k}$ zweier Modulsysteme $\left(\boldsymbol{M}_{1}^{\prime}, \boldsymbol{M}_{2}^{\prime}, \ldots\right),\left(\boldsymbol{M}^{\prime \prime}{ }_{1}, M_{2}^{\prime \prime}, \ldots\right)$ gebildet werden, heisst aus diesen beiden Systemen zusammengesetzt oder componirt, und diese beiden Systeme sollen, wegen der Analogie der Composition mit der Multiplication, auch als Factoren bezeichnet werden.

(') a. a. 0 . S. $77-78 ; 84 ; 85$. Hier findet man die unter Nr. I bis IX gegebenen Sătze; $X$ ist auf S. 85 VII, sowie auf persönliche Mitteilungen seitens des Herrn KRONECKER gegründet. 
Der Ausdruck „Composition” soll ohne Weiteres auf aequivalente Systeme übertragen und demnach auch jedes, dem System $\left(M_{h}^{\prime} M^{\prime \prime}{ }_{k}\right)$ aequivalente System als aus den beiden Systemen $\left(\boldsymbol{M}^{\prime}\right),\left(\boldsymbol{M}^{\prime \prime}\right)$ componirt bezeichnet werden, so dass die Elemente des componirten Systems als bilineare Functionen der beiderseitigen Elemente $M^{\prime}, M^{\prime \prime}$ mit ganzen, dem Bereiche angehörigen Coefficienten zu charakterisiren sind.

IV. Ein Modulsystem heisst irveductibel, wenn es nicht aus zwei anderen zusammengesetzt ist, deren jedes ein Modulsystem im eigentlichen Sinne des Wortes ist.

V. Eine algebraische Form soll als eine andere Form renthaltend) bezeichnet werden, wenn das Coefficientensystem der letzteren in dem der ersteren (nach der in II enthaltenen Bestimmung) enthalten ist.

VI. Ist die Form $F$ in $F_{0}$, aber auch umgekehrt $F_{0}$ in $F$ enthalten, so sind die beiden Formen einander sabsolut aequivalent» (Vgl. II).

VII. Eine Form, welche durch wirkliche Multiplication von zwei anderen Formen entsteht, und jede einem solchen Product aequivalente Form soll eine aus den beiden ersten zusanmengesetzte oder componirte Form genannt werden.

VIII. Eine eigentlich primitive Form ist dadurch charakterisirt, dass ihre Coefficienten keinen Divisor irgend einer Stufe mit einander gemein haben. Jede eigentlich primitive Form ist absolut aequivalent Eins.

IX. Eine Form wird als mnicht zerlegbarn, sirreductibelm oder als „Primforms bezeichnet, wenn sie keinem Producte von zwei nicht primitiven Formen des festgesetzten Bereiches aequivalent ist.

X. Die Aequivalenz wie die Primitivität lässt Abstufungen 7.u: Eine Form ist uneigentlich primitiv zur $k^{\text {ten }}$ Stufe, wenn ihre Coefficienten keine Divisoren von $k^{\text {ter }}$ oder niedrigerer Stufe gemeinsam haben. Zwei Formen sind uneigentlich aequivalent zur $k^{\text {ten }}$ Stufe, wenn sie in allen Divisoren von der ersten bis zur $k^{\text {ten }}$ Stufe übereinstimmen.

Auf diese letzte Definition können wir uns stützen, um die Modnlsysteme in einer unserer Differenzen $y_{i}-y_{k}$, wenigstens so weit es für unsere Zwecke erforderlich ist, zu componiren. Wir benutzen dazu den Begriff der uneigentlichen Aequivalenz der $k^{\text {ten }}$ Stufe; dann dürfen wir einer Form als Factor eine Form, die uneigentlich primitiv von höherer als $\operatorname{der} k^{\text {ten }}$ Stufe ist, hinzufügen, weil dadurch der Charakter der DivisorenSysteme, soweit diese in Frage kommen, nicht geändert wird. 
Beschränkt man sich bei unserem zweiten Beispiele, bei welchem die Notwendigkeit einer allgemeineren Auffassung handgreiflich wurde, auf Aequivalenzen, welche die zweite Stufe nicht überschreiten, so sind für die unbestimmten Grôssen $u_{1}, u_{2}, u_{3}, u_{4}$ die Formen

$$
\left.\psi, \psi \cdot u_{1}\left(x_{2}-x_{3}\right)+u_{2}\left(x_{3}-x_{4}\right)+u_{3}\left(x_{1}-x_{4}\right)+u_{4}\left(x_{2}-x_{3}\right)\right]
$$

einander aequivalent. Das letztere Product ist als homogene, lineare Function der vier Producte

$$
\left(x_{1}-x_{2}\right)\left(x_{1}-x_{4}\right),\left(x_{1}-x_{2}\right)\left(x_{2}-x_{3}\right),\left(x_{1}-x_{4}\right)\left(x_{3}-x_{4}\right),\left(x_{2}-x_{3}\right)\left(x_{3}-x_{4}\right)
$$

darstellbar, so dass hier möglich ist, zu schreiben, was bei absoluter Aequivalenz nicht anging

$$
\psi \equiv 0 \quad \text { modd. }\left[\left(x_{1}-x_{9}, x_{3}-x_{4}\right) \cdot\left(x_{1}-x_{4}, x_{2}-x_{3}\right)\right] .
$$

Dieses Vorgehen lasst sich verallgemeinern.

Die Form $\boldsymbol{F}$ möge zwei Divisoren-Systeme besitzen, von denen keins von höherer als der $k^{\text {en }}$ Stufe sein mag, und auch keins in dem andern enthalten sein soll: $\left(F_{1}, F_{2}, \ldots F_{k}\right),\left(F_{1}^{\prime}, F_{2}^{\prime}, \ldots F_{k}^{\prime}\right)$. Durch diese Voraussetzungen wird bewirkt, dass $k<n$, und dass zweitens

$$
\left(F_{1}, F_{2}, \cdots F_{k}, F_{1}^{\prime}, F_{2}^{\prime \prime}, \cdots F_{k}^{\prime}\right)
$$

von höherer Stufe ist, als jedes der beiden ersteren Systeme. Aus unseren Annahmen folgen die Gleichungen

$$
\begin{aligned}
& F=F_{1} \cdot P_{1}+F_{2} \cdot P_{2}+\cdots+F_{k} \cdot P_{k} \\
& F^{\prime}=F_{1}^{\prime} \cdot P_{1}^{\prime}+F_{2}^{\prime} \cdot P_{2}^{\prime}+\cdots+F_{k}^{\prime} \cdot P_{k}^{\prime} .
\end{aligned}
$$

Fngt man der Function $r$ den Factor

$$
\Phi=u_{\mathrm{i}} F_{1}+u_{2} F_{2}+\cdots+u_{k} F_{k}+u_{1}^{\prime} F_{1}^{\prime}+u_{2}^{\prime} F_{2}^{\prime \prime}+\cdots+u_{k}^{\prime} F_{k}^{\prime \prime}
$$

hinzı, so andert dieser, da er primitiv von $k^{\text {ter }}$ Stufe isţ, die jetzt allein wesentlichen Divisoren-Systeme von $F$ nicht. Nun ist

$$
F^{\prime} \cdot \Phi=\sum_{h=1}^{k} F_{h}^{\prime \prime} \cdot P_{h}^{\prime} \cdot\left[u_{1} F_{1}^{\prime}+\cdots+u_{k} F_{k}\right]+\sum_{h=1}^{k} F_{h} \cdot P_{h}\left[u_{1}^{\prime} F_{1}^{\prime \prime}+\cdots+u_{k}^{\prime} F_{k}^{\prime \prime}\right]
$$

$$
F \cdot \Phi=\sum_{\lambda, \mu} Q_{i^{\prime},} \cdot F_{\lambda} \cdot F_{n}^{\prime}
$$


und daraus erhellt, dass $F^{\prime} . \Phi$ das aus $\left(F_{2}\right),\left(F_{2}^{\prime \prime}\right)$ componirte Divisorensystem $\left(F_{\lambda} F^{\prime \prime}\right)$ besitzt. Aus (5) folgt demnach

$$
\boldsymbol{H}^{\prime} \equiv 0 \quad \text { modd. }\left[\left(\boldsymbol{F}_{\lambda}\right) \cdot\left(\boldsymbol{F}_{\lambda}^{\prime}\right)\right] .
$$

Diese Methode kann angewendet und fortgesetzt werden, so lange Divisorensysteme, die von höherer als der $k^{\text {ten }}$ Stufe sind, für unwesentlich erklärt werden dürfen; es ist dagegen vou einer weiteren Composition mit Systemen, die von gleicher Stufe sind wie $\Phi$, abzusehen.

Für $y_{i}-y_{k}$ ersehen wir die Möglichkeit der Composition aller mit einander nicht identischer Systeme von gleichem Typus, falls wir uns auf uneigentliche Primitivitat der betreffenden Stufe beschränken. Bei identischen Systemen ist eine Composition nicht möglich. Sei etwa, um ein Beispiel hierfür zu geben

$$
\begin{aligned}
G_{1} & =\left[1,\left(x_{1} x_{3}\right)\left(x_{2} x_{4}\right)\right] \\
\sigma_{3} & =\left(x_{1} x_{2} x_{3} x_{4}\right) \\
G_{2}^{\prime} \sigma_{2} & =\left\{\left(x_{1} x_{2} x_{3} x_{4}\right),\left(x_{1} x_{4} x_{3} x_{2}\right)\right\}
\end{aligned}
$$

dann gehört zu jedem $y_{1}-y_{2}$ das Modulsystem $x_{1}-x_{2}, x_{1}-x_{2}$, $x_{1}-x_{4}$. Wählen wir

$$
y_{1}=x_{1}+x_{3}-x_{2}-x_{4}+x_{1} x_{3}+x_{2} x_{4}
$$

so erhellt, dass $y_{1}-y_{2}$ nur jenes Modulsystem, nicht das Quadrat desselben enthălt, weil $y_{i}^{-}-y_{2}$ lediglich Glieder erster Dimension umfasst.

\section{$\S 4$.}

Unsere früheren Untersuchungen zeigten, dass man sămmțtiche Divisorensysteme einer Differenz $y_{i}-y_{k}$, soweit dieselben für die Gattung invariant und also von der Bildung besonderer Functionen $y$ unabhängig sind, aus den Betrachtungen der zugehörigen Gruppe ableiten kann. Diese gewährt hier, wie an vielen anderen Stellen, dieselben Vorteile, welche die KroneCKEn'sche „Fundamentalgleichung der Gattung» bietet.

Ferner sahen wir soeben, wie und in wie weit die Divisorensysteme dessclben Typus von $y_{i}-y_{k}$ eine Composition zulassen. Da endlich eine 
Zusarnmensetzung von Divisorensystemen verschiedener Differenzen $y_{i}-y_{k}$, $y_{l}-y_{m}, \ldots$ gar keine Schwierigkeiten macht, so sind wir in den Stand gesetzt, alle Divisorensysteme eines bestimmten Typus, welche in $D_{y}$ eingehen, zu vereinigen und die Potenz anzugeben, in welcher das componirte System als Modulsystem von $D_{y}$ erscheint.

Es treten hierbei Divisorensysteme von $D_{y}$ auf, deren Elemente rationale ganze Functionen von $\mathfrak{f}_{1}, \mathfrak{f}_{2}, \ldots, \mathfrak{f}_{n}$ sind. Bezeichnen wir ein Divisorensystem von $y_{i}-y_{k}$ mit $\left(u_{1}, v_{1}, w_{1}, \ldots\right)$ und die verschiedenen Werte, welche dasselbe bei den Vertauschungen der $x_{1}, x_{2}, \ldots x_{n}$ untereinander annehmen kann, init $\left(u_{2}, v_{2}, w_{2}, \ldots\right),\left(u_{3}, v_{3}, w_{3}, \ldots\right), \ldots .,\left(u_{u}, v_{u}, w_{u}, \ldots\right), \ldots .$. , so werden alle diese als Divisorensysteme von $D_{y}$ auftreten und, da sie von einander verschieden sein sollen, auch ihr Product. Dies ist jedoch nicht so beschaffen, dass alle seine Elemente symmetrisch in den $x_{1}, x_{2}, \ldots x_{n}$ würden. Un den Schwierigkeiten $z u$ entgehen, welche bei den hierhergehörigen Fragen auftreten und deren directe Behandlung sehr eingehende Untersuchungen zu erfordern scheint, schlagen wir einen anderen Weg ein. Wir bilden Functionensysteme der $\mathfrak{f}_{1}, \mathfrak{f}_{2}, \ldots, \mathfrak{f}_{n}$, deren Gesammtheit nur dann verschwindet, wenn vorgeschriebenc Gleichheiten unter den Wurzeln $x_{1}, x_{2}, \ldots x_{n}$ eintreten, also z. B. nur daun, wenn zwei Paare von Wurzelı oder dann wenn drei Wurzelı einander gleich werden. Wenn nun

$$
\Phi_{1}\left(\mathfrak{f}_{1}, \mathfrak{f}_{2}, \cdots, \mathfrak{f}_{n}\right), \quad \Phi_{2}\left(\mathfrak{f}_{1}, \mathfrak{f}_{2}, \cdots, \tilde{f}_{n}\right), \cdots \quad \Phi_{K}\left(\mathfrak{f}_{1}, \mathfrak{f}_{2}, \cdots, \mathfrak{f}_{n}\right)
$$

ein solches System ist, so liefert es das gesuchte Divisorensystem von $D_{y}$, welches den betreffenden Wurrelgleichheiten angehört, entweder in möglichster Einfachheit oder auch mehrfach. Jedenfalls ist dann, wenm nicht $D_{y}$ selber, so eine Potenz von $D_{y}$ als lineare homogene Function der $\Phi$ mit Coefficienten darstellbar, welche ganz und rational in den $\mathfrak{f}$ sind. Bilden wir mit Beibehaltung der obigen Bezeichungen das symmetrische Product mit den unbestimmten Grössen $\lambda, \mu, \nu, \ldots$

$$
I I\left(u_{\alpha}+\lambda v_{\alpha}+\mu w_{\alpha}+\cdots\right)
$$

und entwickeln dasselbe nach $\lambda, \mu, \ldots$, so geben die Coefficienten eine Reihe von Functionen $\Phi$ unit den gewünschten Eigenschaften, also ein Divisorensystem einer Potenz von $D_{y}$. Schon die bedeutende Anzah] der 
hierbei auftretenden Functionen zeigt, dass das System im Allgemeinen stark reducirt werden kann; die Anzahl der notwendigen Functionen wird höchstens gleich $n+1$ werden. ( $\left.{ }^{1}\right) \mathrm{Zu}$ bemerken ist, dass an die Stelle des Factors $u_{a}+\lambda v_{a}+\mu w_{a}+\ldots$ jeder andere geset $\%$ werden kann, dessen Verschwinden dasjenige von $u_{n}, v_{a}, w_{a}, \ldots$ nach sich zieht; es ist möglich, durch passende Wahl der Elemente von $u_{\alpha}^{\prime}+. \lambda v_{\alpha}^{\prime}+\mu w_{\alpha}^{\prime}+\ldots$ die Anzahl der Factoren zu verringern und damit die Ausführung der Berechnung zu erleichtern. So wird es sich im Allgemeinen empfehlen, nicht die Differenzen $x_{k}-x_{k}$ selbst, sondern ihre Quadrate für $u_{u}, v_{a}, \ldots$ zu benutzen.

Für die Modulsysteme $\left(x_{k}-x_{2}\right)$, welche die einzigen Divisoren erster Stufe sind, ergiebt sich das hekannte Resultat, dass $D_{y}$ die Discriminante $\Delta$ der Grundgleichung

$$
\left(x-x_{1}\right)\left(x-x_{2}\right) \cdots\left(x-x_{n}\right) \equiv x^{n}-\mathfrak{f}_{1} x^{n-1}+\mathfrak{f}_{2} x^{n-2}-\cdots \pm \mathfrak{f}_{n}=0
$$

als Factor enthält, falls (S) nicht symmetrisch ist.

Fur die Modulsysteme zweiter Stufe giebt es zwei verschiedene Typen: einmal kann ein solches durch zwei Paare gleicher Wurzeln, ferner auch durch ein Tripel gleicher Wurzeln hervorgerufen werden.

Im ersten Falle wăre das Product

$$
\Pi\left[\left(x_{1}-x_{8}\right)^{2}+\lambda\left(x_{3}-x_{4}\right)^{2}\right]
$$

im zweiten Falle das Product

$$
I I\left[\left(x_{1}-x_{2}\right)^{2}+\lambda\left(x_{1}-x_{3}\right)^{2}\right]
$$

zu entwickeln. Weil nun aber in diesen. Producten je zwei Factoren einander entsprechen: $\left(u_{u}+\lambda v_{\alpha}\right)$ und $\left(v_{u}+\lambda u_{\alpha}\right)$, und die Multiplication derselben

$$
\left(u_{\alpha} v_{\alpha}+\lambda\left[u_{\alpha}^{2}+v_{\alpha}^{2}\right]+\lambda^{2} u_{\alpha} v_{\alpha}\right)
$$

ergiebt, so kann, da hier wie bei allen Formen nur die Coefficienten von Wichtigkeit sind, das Glied mit $\lambda^{2}$ weggelassen und der Coefficient von $\lambda$ durch $u_{\alpha}+v_{\alpha}$ ersetzt werden; denn

$$
u_{a} v_{a}+\lambda\left[u_{a}+v_{a}\right]
$$

(') Kronecker, a. a. O. S. 37. 
giebt durch sein Verschwinden genau dasselbe, was $\left(Q_{1}\right)$ ergiebt, dass

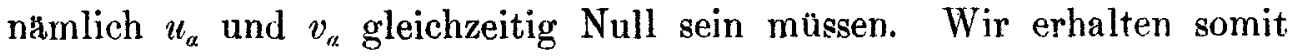

$$
\begin{aligned}
& \Pi\left[\left(x_{1}-x_{2}\right)^{2}\left(x_{3}-x_{4}\right)^{2}+\lambda\left\{\left(x_{1}-x_{2}\right)^{2}+\left(x_{3}-x_{4}\right)^{2}\right\}\right] \\
& \Pi\left[\left(x_{1}-x_{2}\right)^{2}\left(x_{1}-x_{3}\right)^{2}+\lambda\left\{\left(x_{1}-x_{2}\right)^{2}+\left(x_{1}-x_{3}\right)^{2}\right\}\right]
\end{aligned}
$$

Noch einfacher wird die Form durch die Wahl anderer $u_{a}, v_{\alpha}$, z. B.

$$
\begin{aligned}
& \Pi\left[\left(x_{1}+x_{2}-x_{3}-x_{4}\right)^{2}+\lambda\left(x_{1}+x_{3}-x_{2}-x_{4}\right)^{2}\right] \\
& \Pi\left[\left(2 x_{1}-x_{2}-x_{3}\right)^{2}+\lambda\left(2 x_{2}-x_{3}-x_{1}\right)^{2}\right] .
\end{aligned}
$$

Aus dem ersteren kann man für $n=4$ das System ableiten

$$
\begin{aligned}
& \Phi_{1}=\left(x_{1}+x_{2}-x_{3}-x_{4}\right)\left(x_{1}+x_{3}-x_{2}-x_{4}\right)\left(x_{1}+x_{4}-x_{2}-x_{3}\right) \\
& \begin{array}{c}
\Phi_{2}=\left(x_{1}+x_{2}-x_{3}-x_{4}\right)\left(x_{1}+x_{3}-x_{2}-x_{4}\right)+\left(x_{1}+x_{3}-x_{2}-x_{4}\right)\left(x_{1}+x_{4}-x_{2}-x_{3}\right)+ \\
+\left(x_{1}+x_{4}-x_{2}-x_{3}\right)\left(x_{1}+x_{3}-x_{3}-x_{4}\right) ;
\end{array}
\end{aligned}
$$

aus dem zweiten furr $n=3$ das System

$$
\begin{gathered}
\Psi_{1}=\left(2 x_{1}-x_{2}-x_{3}\right)\left(2 x_{2}-x_{3}-x_{1}\right)\left(2 x_{3}-x_{1}-x_{2}\right) \\
\begin{array}{c}
\Psi_{2}=\left(2 x_{1}-x_{2}-x_{3}\right)\left(2 x_{2}-x_{3}-x_{3}\right)+\left(2 x_{2}-x_{3}-x_{1}\right)\left(2 x_{3}-x_{1}-x_{3}\right)+ \\
+\left(2 x_{3}-x_{1}-x_{8}\right)\left(2 x_{1}-x_{2}-x_{3}\right) ;
\end{array}
\end{gathered}
$$

beide können dann für die folgenden Bildungen bei $n>4$ oder $n>3$ benutzt werden:

$$
\begin{aligned}
& \Pi\left[\Phi_{1}+\lambda \Phi_{2}\right] \\
& \Pi\left[\Psi_{1}+\lambda \Psi_{2}\right] .
\end{aligned}
$$

Auf weitere in diese Untersuchung gehörige Verhaltnisse werde ich an einer anderen Stelle genauer eingehen, so dass ich hier davon abbrechen darf. Nur einen Punkt will ich noch hervorheben.

Bilden wir die Gacors'sche Resolventengleichung, welche uuch kurz (entgegen der von SERRET und JornAN benutzten Nomenclatur) als eine Galois'sche Gleichung bezeichnet werden mag, so ist die Gruppe derselben gleich Eins. Die Discriminante $D_{y}$ enthalt deswegen alle überhaupt nur 
möglichen Divisorensysteme und dabei ein jedes in der höchst möglichen Potenz. Wählen wir für die $n$ !-wertige Function $y_{1}$ eine der von $\operatorname{mir}\left({ }^{\prime}\right)$ angegebenen Bildungen mit unbestimmten Exponenten

$$
y_{1}=x_{1}^{\alpha} x_{2}^{\beta} x_{3}^{\gamma} \cdots x_{n}^{s},
$$

dann zeichnen sich die Resultate durch besondere Einfachheit und Ühersichtlichkeit aus. Die Differenzen $y_{i}-y_{k}$ enthalten je zwei Glieder, deren gemeinsame Factoren wcggeworfen werden können. Somit treten unter den Differenzen, welche zu Substitutionen eines bestimmten Typus gehören, verschiedene einfache, charakteristische Bildungen auf, in welche nur die bei den Wurzelgleichheiten $(A ; \S 1)$ erscheinenden $x$ eingehen. So erhalt man in den beiden von uns besprochenen Fällen der Divisorensysteme zweiter Stufe

$$
\begin{array}{ll}
\left(\mathrm{P}_{1} \mathrm{v}\right) & \Pi\left(x_{1}^{m} x_{2}^{n}-x_{3}^{m} x_{4}^{n}\right), \\
\left(\mathrm{P}_{2} \mathrm{IV}\right) & I\left(x_{1}^{m+n}-x_{2}^{m} x_{3}^{n}\right) ;
\end{array}
$$

man erkennt, dass durch das Verschwinden von $n$ solchen Producten Beziehungen von der Form $x_{a}^{\alpha}=x_{a^{\prime}}^{\alpha}$ constituirt werden, und dass demnach eine passend gewahlte $(n+1)^{r e}$ Function desselben Typus hieraus den Schluss $x_{a}=x_{a^{\prime}}$ u. s. w. ermöglicht.

Die Potenz, in welcher ein Divisorensystem, falls es das darzustellende Gebilde einfach liefert, als Factor von $D_{y}$ auftritt, ist aus der Betrachtung der Gruppe leicht $\mathrm{zu}$ bestimmen, sobald man die gesammte Tabelle (C) als vorliegend ansieht; dagegen wird die Feststellung der Anzahl schwieriger, wenn nur die erste Zeile von (C) nämlich $G_{1}$ als bekannt gilt. Für den einfachsten Fall, für den Factor $\Delta$, ergiebt sich jedoch auch hier sofort als Exponent

$$
\rho\left[\frac{1}{2} n(n-1)-q\right]
$$

für den Fall von drei einander gleichen Wurzeln habe ich die Frage gleichfalls erledigt; $\left({ }^{2}\right)$ es lohnt sich jedoch nicht auf weitere Untersuchungen derselben einzugehen.

(') Substitutionentheorie. \& 31.

(') Journal f. reine u. angewandte Mathematik. B. XC; S. 164, ff. 


\section{$\S \mathbf{5}$}

Waren wir statt von einer allgemeinen von einer speciellen Gleichung $n^{\text {ten }}$ Grades ausgegangen, so häte es sich ereignen können, dass einige oder auch wohl alle Gattungen ganzer Functionen der Wurzeln einen gemeinsamen Teiler, der vorher bei allgemeinen Gleichungen constatirt wurde, nicht mehr besitzen. Diesen Fall wollen wir eingehender untersuchen. Um von der allgemeinen Gleichung

$$
x^{n}-\mathfrak{f}_{1} x^{n-1}+\mathfrak{f}_{2} x^{n-2}-\cdots \pm \mathfrak{f}_{n} \equiv\left(x-x_{1}\right)\left(x-x_{2}\right) \cdots\left(x-x_{n}\right)=0
$$

zu einer beliebigen speciellen zu kommen, ist die Adjungirung einer einzigen Gattung $\mathscr{B}_{1}$ ihrer Wurzeln notwendig und hinreichend. Die so modificirte Gleichung wollen wir mit $\left(l^{\prime}\right)$ bezeichnen. Genau genommen kommen wir dabei aber nicht zu einer einzigen, sonderm zu einer ganzen Classe von Gleichungen, und von einer solchen gelten die nachstehenden Untersuchungen. Substitutionentheoretisch hat die Adjungirung den Effect, dass bei allen weiteren an (1') geknüpften Betrachtungen nur diejenigen Substitutionen angewendet werden dürfen, welche zu der Gruppe $G_{1}$ von $\mathfrak{S}_{1}$ gehören, da jede $z u \mathbb{S}_{1}$ gehörige Function $y_{1}$ ungeändert bleiben muss. Wahlt man als zu behandelnde Gattung der Wurzeln $x_{1}, x_{2}, \ldots, x_{n}$ von (1') die Gattung $\Gamma_{1}^{\prime}$, so gehoren zu dieser in dem Bereiche $\left(\mathscr{G}_{1}, \mathfrak{f}_{1}, \ldots, \mathfrak{f}_{n}\right)$ nur diejenigen Substitutionen der Gruppe von $\Gamma_{1}^{\prime}$, welche gleichzeitig in $\mathbb{B}_{1}$ vorkommen. Wir kőnnen daher in diesem Falle $I^{\prime \prime}{ }_{1}$ durch $\mathbb{S}_{1}^{\prime}=\Gamma_{1}+u \mathscr{G}_{1}$ ersetzen und bewirken hierdurch, dass die Gruppe $G_{1}^{\prime}$ von $B^{\prime}{ }_{1}$ eine Untergruppe von $G_{1}$ wird. Der Quotient der Ordnungen von $G_{1}$ und $G_{1}^{\prime}$ sei $\rho$; dann besitzt jede Function $y_{1}^{\prime}$ der Gattung $\left(\mathscr{S}_{1}^{\prime}\right.$ innerhalb des Bereiches $\left(\mathscr{G}_{1} ; \mathfrak{f}_{1}, \mathfrak{f}_{2}, \ldots, \mathfrak{f}_{n}\right)$ gerade $\rho$ conjugirte Werte $y_{1}^{\prime}, y_{2}^{\prime}, \ldots \mathscr{y}_{\rho}^{\prime}$, und der im zweiten Paragrafen aufgestellten Tabelle $(\mathrm{C})$ entspricht hier eine Tabelle $\left(\mathrm{C}^{\prime}\right)$, welche in $\rho$ Zeilen alle Substitutionen von $G_{1}$ und zwar in der ersten derselben alle Substitutionen von $G^{\prime}{ }_{1}$ enthält.

Die Untersuchungen des ersten Abschnittes zeigen, dass die Discriminanten $D_{y^{\prime}}$ aller Functionen $y^{\prime}$ der Gattung (S) $^{\prime}$ nur dann einen gemeinsamen Teiler, eine 》Gattungsdiscriminante» besitzen, $\left({ }^{1}\right)$ wenn es Transposi-

( ${ }^{1}$ ) Von gemeinsamen Zahlenfactoren ist abgesehen. Alle Kreisteilungsgattungen besitzen durch $p$ teilbare Discriminanten; diese gelten nicht als Gattungsdiscriminanten im eigentlichen Sinne. 
tionen giebt, welche in $G_{1}$ aber nicht in $G_{1}^{\prime}$ vorkommen, also in $\left\langle\mathrm{C}^{\prime}\right\rangle$ ausserhalb der ersten Zeile.

Haben wir uns demnach die Aufgabe zur Lösung vorgelegt: Es sollen alle Gattungen $\mathbb{B}_{1}$ gefunden werden, für welche es Gattungen ohne Gattungsdiscriminante giebt, - so lisst sich diese jetzt auch folgendermassen formuliren: Es sollen alle Gruppen $G_{1}$ gefunden werden, bei denen gewisse Untergruppen $G_{1}^{\prime}$ genau dieselben Transpositionen umfassen wie $G_{1}$.

Wir teilen hier die Untersuchung, um einen ersichtlichen Fall sofort zu erledigen. $G_{1}$ möge ïberhaupt keine Transpositionen umfassen. Dann enthält keine Grattung der Wurzeln einer hierdurch bestimmten Gleichung eine Gattungsdiscriminante. Und umgekehrt: Wenn keine Gattung der Wurzeln einer $z u\left(\mathfrak{S}_{1} ; \mathfrak{f}_{1}, \mathfrak{f}_{2}, \ldots, \mathfrak{f}_{n}\right)$ gehörigen Gleichung eine Gattungsdiscriminante besitzt, dann kommen in $G_{1}$ keine Transpositionen vor. Der einfachste Fall dieser Art ist der, in welchem $\mathscr{G}_{1}$ die alternirende oder eine unter ihr enthaltene Gattung darstellt; dasselbe ereignet sich bei der cyklischen, der metacyklischen, der halbmetacyklischen Gattung; ferner bei jeder primitiven Gruppe, deren Ordnung $<n$ ! ist, u. s. f.

Zweitens betrachten wir den Fall, dass $G_{1}$ Transpositionen besitzt. Kommen diese nicht sämmtlich in $G^{\prime}{ }_{1}$ vor, so treten in die Discriminante $D_{y^{\prime}}$ als Factor einer oder mehrere der in $\left(\mathscr{G}_{1} ; \mathfrak{f}_{1}, \mathfrak{f}_{2}, \ldots\right)$ irreductiblen Teiler, in welche $\Delta$ bei der Adjungirung irgend einer nicht symmetrischen Gruppe $G_{1}$ zerlegt wird. Wäre nämlich $\Delta$ nach der Adjungirung irreductibel geblieben, so gabe es in $G_{1}$ eine Substitution, welche $\left(x_{a}-x_{\beta}\right)^{2}$ in $\left(x_{\gamma}-x_{i}\right)^{2}$ unwandelt, wobei $\alpha, \beta ; \gamma, \partial$ beliebige Indices der Reihe $1,2, \ldots \ldots, n$ sind; diese Substitution sei $t=\ldots x_{\alpha} x_{\gamma} \ldots x_{\beta} x_{j} \ldots$ Ist jetzt $\sigma=\left(x_{u} x_{\beta}\right)$ eine von den der Voraussetzung gemäss vorhandenen Transpositionen von $G_{1}$, so enthält diese Gruppe auch $t^{-1} \sigma t=\left(x_{\gamma} x_{\partial}\right), \mathrm{d}$. h. alle I'ranspositionen, und sie wäre sonach gegen die Annahme mit der symmetrischen Gruppe identisch. Potenzen solcher Factoren von $\Delta$ übernehmen hier also die Rolle der Gattungsdiscriminante.

Wir fragen weiter, unter welchen Bedingungen, in dem Falle dass $G_{1}$ Transpositionen besitzt, genau dieselben auch in $G_{1}^{\prime}$ vorkommen, wann also für $\mathscr{B}_{1}{ }_{1}$ keine Gattungsdiscriminante besteht. Wir wollen bei der Untersuchung hierüber annehmen, dass (1') irreductibel sei; wir beschränken uns also, nach der Kronecken'schen Terminologie, auf die Adjungi- 
rung seigentlicher Gattungen); nach der $\mathrm{C}_{\Delta \mathrm{UCHY}}$ 'schen auf die stransitiver Gruppen)\%.

Danı lässt sich sofort constatiren, dass $G_{1}$ eine imprimitive Gruppe ist. Denn eine primitive Gruppe, welche Transpositionen besitzt, wie dies bei $G_{1}$ zutrifft, muss symmetrisch sein. (Vgl. Substitutionentheorie $\S 74$ ).

Aus diesem ersten Resultate folgt weiter (ibid. § 222), dass (1') das Resultut der Elimination einer Grösse $x^{\prime \prime}$ (us zwei algebraischen Gleichungen ist:

$$
y_{1}\left(x ; x^{\prime \prime} ; \mathfrak{f}_{1}, \mathfrak{f}_{2}, \cdots, \mathfrak{f}_{n}\right)=0, \quad g^{\prime \prime}\left(x^{\prime \prime} ; \mathfrak{f}_{1}, \mathfrak{f}_{2}, \cdots, \mathfrak{f}_{n}\right)=0
$$

Sollte $g_{1}\left(x, x^{\prime \prime}\right)=0$ in Gebiete $\left(x^{\prime \prime} ; \mathfrak{f}_{1}, \mathfrak{f}_{2}, \ldots\right)$ noch imprimitiv sein, so kann man diese Gleichung als Eliminationsresultat von $x^{\prime}$ aus den beiden Gleichungen

$$
g\left(x ; x^{\prime} ; x^{\prime \prime} ; \mathfrak{i}_{1}, \cdots\right)=0, \quad g^{\prime}\left(x^{\prime} ; x^{\prime \prime} ; \mathfrak{f}_{1}, \cdots\right)=0
$$

auffiassen. Wir wollen, un den Abschluss, der sich ja einstellen muss, sofort herbeizuführen, g als primitiv ansehen. Dann erscheint (1') als lesultat der Elimination von $x^{\prime}, x^{\prime \prime}$ aus

$$
y\left(x ; x^{\prime} ; x^{\prime \prime} ; \mathfrak{f}_{1}, \cdots\right)=0, \quad g^{\prime}\left(x^{\prime} ; x^{\prime \prime} ; \mathfrak{f}_{1}, \cdots\right)=0, \quad y^{\prime \prime}\left(x^{\prime \prime} ; \mathfrak{f}_{1}, \cdots\right)=0
$$

in der form

$$
\prod_{x=1}^{k} \prod_{\beta=1}^{\lambda} y\left(x ; x_{a, \beta}^{\prime} ; x_{a}^{\prime \prime} ; \mathfrak{f}_{i}, \cdots\right)=0
$$

Hierbei bedeuten $x^{\prime \prime}{ }_{1}, x_{2}^{\prime \prime}{ }_{2} ; \ldots . x^{\prime \prime \prime}$ sammtiche Wurceln von $g^{\prime \prime}\left(x^{\prime \prime}\right)=0$; ferner $x^{\prime}{ }_{a 1}, x^{\prime}{ }_{k 2}, \ldots x_{a k}^{\prime}$ sümmitliche Wurzeln von $g^{\prime}\left(x^{\prime} ; x^{\prime \prime}{ }_{a}\right)=0$. Endlich wollen wir mit $x_{a, \beta 1}, x_{\alpha, \beta,}, \ldots x_{a, \beta, n}$ die Wurzcln von $g\left(x ; x_{a, \beta}^{\prime} ; x^{\prime \prime}{ }_{a}\right)=0$ bezeichnen.

Dic zu (1') resp. (4) gehörigen Substitutionen ordnen wir nunmelır in cine Tabelle ein, deren erste Zeile alle diejenigen enthalten soll, durch welche die beiden ersten Indices nicht berührt werden; die einzelnen Cyklen sind bei ihwen von der Form $\left(x_{u \hat{\beta} h t} x_{\alpha_{\beta} \xi k} \ldots x_{u_{j \beta \xi} m}\right)$. Diese Substitutionen werden erlangt, wenn man die $k \cdot \lambda$ einander ähnlichen Gruppen aufstellt, welche zu den Gleichungen

$$
g\left(x ; x_{a, j}^{\prime} ; x^{\prime \prime} ; \mathfrak{i}_{1}, \cdots\right)=0 \quad(\alpha=1,2, \cdots k ; \beta=1,2, \cdots \lambda)
$$

gehören; diese seien $I_{11}^{\prime}, I_{12}, \ldots, I_{a,\{}^{\prime}, \ldots$ Die erste Zeile unserer Tabelle enthält dunn die Gruppe $\left(I_{11}, l_{12}, \ldots I_{\alpha \hat{\beta}}, \ldots\right)=H$. 
Um die zweite Zeile zu bilden multipliciren wir $H$ mit irgend einer nicht der ersten Zeile enthaltenen Substitution. In Folge der Imprimitivität vertauscht diese Substitution Systeme von Wurzeln, welche durch die beiden ersten Indices charakterisirt werden; dieselbe Vertauschung der Systeme findet dann für die ganze Zeile statt. Hieraus ist zu ersehen, dass nur die erste Zeile der Tabelle Transpositionen der Wurzeln $x$ enthalten kann. Die einander ähnlichen Gruppen $\Gamma_{11}, \ldots, \Gamma_{\mu \nu}$ müssen somit Transpositionen besitzen; sie sind primitiv, folglich sind sie auch symmetrisch: demnach ist $g(x)=0$ eine allgemeine Gleichung.

Nach dieser Zurechtlegung der imprimitiven Gruppe $G_{i}$ kehren wir zu den obigen Untersuchungen zurück und ersehen jetzt ohne Schwierigkeit das Schlussresultat. $G_{1}^{\prime}$ muss alle Transpositionen der ersten Zeile, d. h. die gesammte erste Zeile selbst umfassen; enthălt $G^{\prime}$ ferner eine Substitution irgend einer Zeile der 'Tabelle, so umschliesst $G^{\prime}{ }_{1}$ die ganze Zeile; $G_{1}^{\prime}$ soll eine Untergruppe von $G_{1}$ sein und darf demnach nicht alle Zeilen der Tabelle enthalten. Das heisst:

Um sämmtliche Gruppen $G_{1}$ aufzustellen, welche mit gewissen Untergruppen $G^{\prime}$ alle Transpositionen gemeinsam haben, bildet man eine Reihe von irreductiblen Gleichungen

$$
\begin{aligned}
g\left(x, x^{\prime}, x^{\prime \prime}, \cdots, x^{(\nu)} ; \mathfrak{f}_{1}, \cdots, \mathfrak{f}_{n}\right) & =0, & g^{\prime}\left(x^{\prime}, x^{\prime \prime}, \cdots, x^{(\nu)} ; \mathfrak{f}_{1}, \cdots, \mathfrak{f}_{n}\right) & =0 \\
g^{\prime \prime}\left(x^{\prime \prime}, \cdots, x^{(\nu)} ; \mathfrak{f}_{1}, \cdots, \mathfrak{f}_{n}\right) & =0, \cdots & g^{(\nu)}\left(x^{(\nu)} ; \mathfrak{f}_{1}, \cdots, \mathfrak{f}_{n}\right) & =0
\end{aligned}
$$

deren eiste eine allgemeine Gleichung in $x$ mit den willkürlichen Parametern $x^{\prime}, x^{\prime \prime}, \ldots, x^{(\nu)}$ ist, eliminirt aus ihnen diese Parameter und bildet $G_{1}$ als Gruppe der resultirenden Gleichung; $G_{1}^{\prime}$ erhält man, wenn man den Gleichungen $g^{\prime}=0, g^{\prime \prime}=0, \ldots, g^{(\nu)}=0$ beliebige Gattungen ihrer Wurzeln adjungirt. Der $\S 73$ meiner Substitutionentheorie zeigt, wie man auf rein sub. stitutionentheoretischem Wege die fraglichen Gruppen construiren kann. Das einfachste Beispiel bietet sich bei den Gleichungen vierten Grades dar. Nimmt man $g$ und $g^{\prime}$ vom zweiten Grade, so findet sich das Resultat: Jede durch Quadratwurzeln lösbare Gleichung vierten Grades besitzt eine Gattung vierwertiger Functionen der Wurzeln, für welche keine Gattungsdiscriminante besteht. Es ist dieser Ausspruch eine Übersetzung des Satzes, dass alle Transpositionen der Gruppe

$$
G_{1}=\left[1,\left(x_{1} x_{2}\right),\left(x_{3} x_{4}\right),\left(x_{1} x_{2}\right)\left(x_{3} x_{4}\right),\left(x_{1} x_{3}\right)\left(x_{2} x_{4}\right),\left(x_{1} x_{4}\right)\left(x_{2} x_{3}\right),\left(x_{1} x_{3} x_{2} x_{4}\right),\left(x_{1} x_{4} x_{2} x_{3}\right)\right]
$$


auch in der Untergruppe

$$
G_{1}^{\prime}=\left[1,\left(x_{1} x_{2}\right),\left(x_{3} x_{4}\right),\left(x_{1} x_{2}\right)\left(x_{3} x_{4}\right)\right]
$$

auftreten. Weitere Beispiele lassen sich leicht bilden.

\section{$\S 6$.}

Alle die Auseinandersetzungen des vorigen Paragrafen, welche sich auf Transpositionen bezogen und in ihren Resultaten Eigenschaften der Gattungsdiscriminanten nachwiesen, können ohne besondere Modificationen auf den Fall erweitert werden, dass wir Substitutionen von bestimmten anderen Typen betrachten. So sieht man z. B.: Enthält $G_{1}$ ïberhaupt keine Substitutionen des Typus

$$
\left(x_{1} x_{2} x_{3}\right) \text { oder }\left(x_{1} x_{2}\right)\left(x_{3} x_{4}\right)
$$

so besitzt keine Gattung $\mathscr{S}^{\prime}{ }_{1}$ von Wurzeln einer zur Classe $\left(\mathbb{S}_{1} ; \mathfrak{f}_{1}, \mathfrak{f}_{2}, \ldots, \mathfrak{f}_{n}\right)$ gehörigen Gleichung Divisorensysteme zweiter Stufe, welche den Discriminanten $D_{y_{1}^{\prime}}$ aller Functionen $y_{1}^{\prime}$ der Gattung gemeinsam wären.

Kommen alle Substitutionen eines bestimmten Typus, welche in $G_{1}$ enthalten sind, auch in der Untergruppe $G_{1}^{\prime}$ vor, so besitzen die Discriminanten von Functionen der Gattung ${ }^{0}{ }^{\prime}{ }_{1}$ kein Divisorensystem gemeinsam, welches aus den Substitutionen jenes Typus entspringt.

Ein allgemeines hierher gehöriges Theorem leiten wir auf folgende Art ab: Wir verstehen unter $\gamma$ eine zur GaLors'schen Gattung gehörige Function bei einer allgemeinen Gleichung $n^{\text {ten }}$ Grades, so dass für $r$ die Maximalzahl von Werten $n !=N$ besteht. Auf die Reihe

$$
r_{1}, r_{2}, r_{3}, \cdots, r_{N}
$$

dieser Werte wird die Gruppe sümmtlicher Substitutionen von $x_{1}, x_{\dot{2}}, \ldots, x_{n}$ angewendet, und jede der hierdurch hervorgerufenen Gruppirungen der $r$ als eine Permutation der Elemente $r$ gegenüber der Anordnung $(I)$ betrachtet. Die so entstehenden Substitutionen bilden eine Gruppe $\Sigma$ der Ordnung $N=n$ !, welche der Gruppe $S$ aller Substitutionen unter den $x$ einstufig isomorf ist. Jede Substitution von $\Sigma$ setzt alle Elemente $\gamma$ um; ferner ist eine jēde regulär (Vgl. Substitutionentheorie $\$ 89$ ). Die Sub- 
stitutionen, welche Divisorensysteme von möglichst niedriger Stufe hervorrufen, haben demnach die Form $\left(r_{1} r_{2}\right)\left(r_{3} r_{4}\right) \ldots\left(r_{N-1} r_{N}\right)$ und die Divisorensysteme sind somit von der Stufe $\frac{1}{2} N=\frac{1}{2} n$ !.

Es giebt Classen von Gleichungen der Grade n!, deren Gattungen als Divisorensysteme der Discriminanten nur solche der Stufe $\frac{1}{2} n$ ! oder solche von höherer Stufe besitzen.

Als Beispiel wăhlen wir den Fall $n=3$. Dann wird $\Sigma$ die Substitutionen

$$
\begin{aligned}
& 1,\left(\gamma_{1} \gamma_{2}\right)\left(\gamma_{3} \gamma_{4}\right)\left(\gamma_{5} \gamma_{6}\right),\left(r_{1} \gamma_{4}\right)\left(\gamma_{2} \gamma_{5}\right)\left(\gamma_{3} \gamma_{6}\right),\left(\gamma_{1} \gamma_{6}\right)\left(\gamma_{2} \gamma_{3}\right)\left(\gamma_{4} \gamma_{5}\right) \\
& \left(r_{1} r_{3} r_{5}\right)\left(r_{2} \gamma_{6} r_{4}\right),\left(r_{1} r_{5} r_{3}\right)\left(r_{2} r_{4} r_{6}\right)
\end{aligned}
$$

enthalten; setzt man $\gamma=x_{1} x_{2}{ }^{2}$ und nimmt man

$$
x^{3}+c_{2} x-c_{3}=0
$$

als diejenige Gleichung, deren Wurzeln $x_{1}, x_{2}, x_{3}$ sind, so wird die Gleichung sechsten Grades für $\gamma$ die folgende sein:

$\gamma^{6}+3 c_{3} \gamma^{5}+\left(6 c_{3}{ }^{2}+c_{2}{ }^{3}\right) \gamma^{4}+\left(7 c_{3}{ }^{3}+2 c_{2}{ }^{3} c_{3}\right) \gamma^{3}+c_{3}{ }^{2}\left(6 c_{3}{ }^{2}+c_{2}{ }^{3}\right) \gamma^{2}+3 c_{3}{ }^{5} \gamma+c_{3}{ }^{6}=0$.

Sie hat also die Eigenschaft, dass die Discriminante jeder beliebigen Function ihrer Wurzeln von Divisorensystemen erster und zweiter Stufe frei ist.

Die nachstehende zweite Methode führt zu ähnlichen Resultaten. Wir nehmen $y_{1}$ als eine beliebige Funetion $\rho^{t e r}$ Ordnung, welche $z u$ der beliebigen Gruppe $G_{1}$ gehören möge. Die $\rho$ Werte von $y_{1}$ sollen

$$
y_{1}, y_{2}, y_{3}, \cdots, y_{\rho}
$$

sein. Wir setzen voraus, dass $\rho>2$ angenornmen werde. Auf die Reihe (Y) wenden wir alle Substitutionen der alternirenden Gruppe $\mathfrak{H}$ von $x_{1}, x_{2}, \ldots, x_{n}$ an. Ist $G_{1}$ eine Untergruppe von $\mathfrak{A}$, so liefert die Anwendung von $\mathfrak{A}$ auf $y_{1}$ nur die Hälfte aller Werte dieser Function

$$
y_{1}, y_{2}, y_{3}, \cdots, y_{\frac{\rho}{2}}
$$

ist $G_{1}$ dagegen nicht in der alternirenden Gruppe enthalten, so bekommen wir aus $y_{1}$ alle $\rho$ Werte $(\mathrm{Y})$. Je nachdem der eine oder der andere dieser beiden Falle eintritt, erhalten wir durch die Anwendung volı $\mathfrak{I}$ 
auf $\left(\mathrm{Y}^{\prime}\right)$ resp. $(\mathrm{Y})$ eine transitive Gruppe $A$ von $\frac{\rho}{2}$ oder von $\rho$ Elementen. Es ist $A$ von der Ordnung $\frac{1}{2} n$ ! und einstufig isomorf zu $\mathfrak{A}$; denn zwei von einander verschiedene Substitutionen von $\mathfrak{A}$ müssen verschiedene Sub. stitutionen von $A$ hervorrufen. Weil ferner $\mathfrak{A}$ einfach ist, so kann auch $A$ nicht zusammengesetzt sein. (Substitutionentheorie $§ 88$.)

Wir unterscheiden nun die beiden Fälle, ob $A$ primitiv oder imprimitiv ist. Im ersteren darf; falls $\rho$ eine gewisse von $p$ abhangige Grenze überschreitet, $A$ keine cyklische Substitution einer Primzahlordnung enthalten, welche kleiner oder gleich $p$ ist: denn sonst ginge (a. a. O. $\$ 74 \mathrm{ff}$.) $A$ in die alternirende oder die symmetrische Gruppe der $\frac{\rho}{2}$ resp. $\rho$ Elemente $y_{1}, y_{2}, \ldots, y_{\rho}$ über, was wegen der bekannten Sätze über die Grösse von $\rho$ in Beziehung auf $n$ nicht möglich ist.

Hinsichtlich des Falles einer imprimitiven Gruppe $A$ erinnern wir uns an einen Satz (a. a. O. $\$ 84$, Zusatz II), welcher in der correcten Fassung folgendermassen lautet: Jede imprimitive Gruppe ist zusammengesetzt, falls sie von der Einheit verschiedene Substitutionen besitzt, welche die einzelnen Systeme der Imprimitivitat ungeändert lassen. Nun ist $A$ einfach, und somit darf diese Gruppe keinc Substitutionen enthalten, welche lediglich die Elemente der einzelnen Systeme unter cinander umsetzen. Es dürfen demnach in $A$ nberhaupt keine cyklischen Substitutionen von Primzahlordnung vorkommen, weil diese gar nicht Elemente verschiedener Systeme enthalten können. Hicraus erhcllt: Die Classe der durch $A$ charakterisirten Gleichungen des Grades $\frac{1}{2} \rho$ oder $\rho$ und der Ordnung $\frac{1}{2} n$ ! enthält nur solche Gattungen von Wurzeln, deren Discriminanten leine Divisorensysteme besitzen, welche aus einer cyllischen Substitution der Primahlordnung $q<p$ entspringen; $p$ ist dabei eine von o abhängige Zahl, die mindestens den Wert is hat.

Dass wir bei der Durchführung ahnlicher Untersuchungen zu ähnlichen liesultaten gelangen können, mag folgendes Beispiel zeigen.

Die Wurzeln von

$$
a^{4}-c_{1} x^{3}+c_{2} x^{2}-c_{3} x+c_{4}=0
$$

seien $x_{1}, x_{2}, x_{3}, x_{4}$. Wir setzen

$$
y_{1}=x_{1} x_{3}, y_{3}=x_{1} x_{3}, y_{3}=x_{1} x_{4}, y_{4}=x_{2} x_{3}, y_{5}=x_{2} x_{4}, y_{6}=x_{3} x_{4}
$$


und wenden auf die $x$ die symmetrische Gruppe dieser vier Elemente an. Dadurch werden 24 Substitutionen unter den $y_{1}, y_{2}, \ldots, y_{6}$ hervorgerufen, welche eine zur symmetrischen Gruppe $S$ von $x_{1}, \ldots, x_{4}$ einstufig isomorfe Gruppe $\Sigma$ bilden. Diese umfasst die folgenden Substitutionen

$$
\begin{array}{r}
1, \quad\left(y_{1} y_{2}\right)\left(y_{5} y_{6}\right),\left(y_{1} y_{3}\right)\left(y_{4} y_{6}\right) ;\left(y_{1} y_{4}\right)\left(y_{3} y_{6}\right),\left(y_{1} y_{5}\right)\left(y_{2} y_{6}\right), \\
\left(y_{1} y_{6}\right)\left(y_{2} y_{5}\right),\left(y_{1} y_{6}\right)\left(y_{3} y_{4}\right),\left(y_{2} y_{3}\right)\left(y_{4} y_{5}\right),\left(y_{2} y_{4}\right)\left(y_{3} y_{5}\right),\left(y_{2} y_{5}\right)\left(y_{3} y_{4}\right), \\
\left(y_{1} y_{4} y_{3}\right)\left(y_{4} y_{5} y_{6}\right),\left(y_{1} y_{3} y_{2}\right)\left(y_{4} y_{6} y_{5}\right),\left(y_{1} y_{2} y_{4}\right)\left(y_{3} y_{6} y_{5}\right),\left(y_{1} y_{4} y_{2}\right)\left(y_{3} y_{5} y_{6}\right), \\
\left(y_{1} y_{3} y_{5}\right)\left(y_{2} y_{6} y_{4}\right),\left(y_{1} y_{5} y_{3}\right)\left(y_{2} y_{4} y_{6}\right),\left(y_{1} y_{4} y_{5}\right)\left(y_{2} y_{6} y_{9}\right),\left(y_{1} y_{5} y_{4}\right)\left(y_{2} y_{3} y_{6}\right), \\
\left(y_{1} y_{2} y_{6} y_{5}\right)\left(y_{3} y_{4}\right),\left(y_{1} y_{5} y_{6} y_{2}\right)\left(y_{3} y_{4}\right),\left(y_{1} y_{3} y_{6} y_{4}\right)\left(y_{2} y_{5}\right), \\
\left(y_{1} y_{4} y_{6} y_{3}\right)\left(y_{2} y_{5}\right),\left(y_{2} y_{3} y_{5} y_{4}\right)\left(y_{1} y_{6}\right),\left(y_{1} y_{4} y_{6} y_{3}\right)\left(y_{2} y_{5}\right)
\end{array}
$$

Dic Gleichung sechsten Grades, derer Wurzeln $y_{1}, y_{2}, \ldots, y_{6}$ sind, lautet

$$
\begin{gathered}
y^{6}-3 c_{1} y^{5}+\left(2 c_{2}+3 c_{1}{ }^{2}\right) y^{4}+\left(4 c_{1} c_{2}+c_{1}{ }^{3}\right) y^{3}+\left(-4 c_{4}+c_{3} c_{1}+c_{2}{ }^{2}+2 c_{2} c_{1}{ }^{2}\right) y^{2} \\
-\left(30 c_{5}+4 c_{4} c_{1}-c_{3} c_{1}{ }^{2}\right) y+\left(32 c_{6}-7 c_{5} c_{1}-c_{3}{ }^{2}-c_{4} c_{1}{ }^{2}+c_{3} c_{2} c_{1}\right)=0
\end{gathered}
$$

die Gruppe derselben ist У'. Aus der Beschaffenheit ihrer Substitutionen geht hervor, dass keine Gattung der Wurzeln $y$ existirt, für welche die Discriminanten der zugehörigen Functionen einen Teiler oder auch ein Divisorensystem dritter oder fünfter Stufe gemeinsam hätten.

\section{$\$ 7$.}

Für die nun folgenden allgemeineren Entwickelungen sind einige teils bekannte, teils leicht zu beweisende Hülfssătze nötig, welche hier zusammengestellt werden sollen:

I. Gehören zu einer Gruppe $G$ alle Substitutionen eines bestimmten beliebigen Typus, so ist $G$ entweder die alternirende oder die symmetrische Gruppe.

II. Jede Gruppe, welche nicht unter der alternirenden steht, ist zusammengesetzt; diejenigen ihrer Substitutionen, welche in der alternirenden Gruppe vorkommen, bilden eine ausqezeichnete Untergruone mit 2 als Factor der Zusammensetzung. 
III. Wendet man auf eine beliebige Function $\varphi\left(x_{1}, x_{2}, \ldots, x_{n}\right)$ alle Substitutionen $\operatorname{der} x$ an, so entsteht unter den $\rho$ Werten $\varphi_{1}, \varphi_{2}, \ldots, \varphi_{\rho}$ eine transitive Gruppe.

IV. Entsprechende Substitutionen isomorfer Gruppen besitzen gleiche Ordnung.

V. Ist eine Gruppe einfach oder zusammengesetzt, so ist jede ihr isomorfe Gruppe gleichfalls entsprechend einfach oder zusammengesetzt.

VI. Jede isomorfe transitive Gruppe $I$ von $G$ entspricht einer Untergruppe $H$ von $G$ derart, dass der Grad von $I^{\prime}$ gleich dem Quotienten aus den Ordnungen von $G$ und $H$ ist. Ist also z. B. $G$ die alternirende Gruppe von $x_{1}, x_{2}, \ldots, x_{n}$ so ist $r$ von einem der Grade $1, n, n(n-1), \ldots$

Wir bezeichnen nun durch $\mathfrak{A}$ und $\subseteq$ die alternirende und die symmetrische Gruppe der $n$ Elemente $x_{1}, x_{2}, \ldots, x_{n}$. Es seien $\varphi_{1}, \varphi_{2}, \ldots, \varphi_{\rho}$ die $\rho$ Werte einer rationalen ganzen Function $\varphi\left(x_{1}, \ldots, x_{n}\right)$. Wenden wir alle Substitutionen von $\mathfrak{P}$ oder von $\subseteq$ auf die Reihe $\varphi_{1}, \varphi_{2}, \ldots, \varphi_{p}$ an, dann kann man die hervorgerufenen Umstellungen als Substitutionen unter den $\varphi$ deuten; die so entstehenden Gruppen seien entsprechend $A$ oder 2 ; 2 ist transitiv in den $\rho$ Werten $\varphi$ (Hülfss. III). Käme in $\Sigma$ eine Transposition $\tau=\left(\varphi_{a} \varphi_{\beta}\right)$ vor und diese entspräche (Halfss. IV) der Substitution zweiter Ordnung $t=\left(x_{a} x_{b}\right)\left(x_{c} x_{d}\right) \ldots$ in $\varsigma_{\text {, dann wäden allen }}$ Substitutionen $t, t^{\prime}, t^{\prime \prime}, \ldots$ in $S$, welche von demselben Typus sind wie $t$, in 2 Transpositionen $\tau, \tau^{\prime}, \tau^{\prime \prime}, \ldots$ entsprechen, da die Operationen $s^{-1} t s$ und $\sigma^{-1} \tau \sigma$ einander entsprechen. Ebenso würden forner die Gruppen $G=\left\{t, t^{\prime}, t^{\prime \prime}, \ldots\right\}$ und $l^{\prime}=\left\{\tau, \tau^{\prime}, \tau^{\prime \prime}, \ldots\right\}$ einander isomorf sein. Gernäss Hulfss. I ist $G$ alternirend oder symmetrisch, also $I$ entweder gleich $A$ oder gleich 2 ; da $I$ die Transposition $\left(\varphi_{a} \varphi_{\beta}\right)$ enthalt, ist sie zusammengesetzt (Hülfss. II); demnach gilt dasselbe von $G$ (Hülfss. V); also ist $G=S$ und $I^{\prime}=\Sigma$. Sobald eine transitive Gruppe $I^{\prime}$ eine 'Transposition enthält, ist sie symmetrisch, und sonach von der Ordnung $\rho$ !. Wir haben also $\rho !=n !$, da 2 , $S$ einstufig isomorf sind; folglich ist $\rho=n$, d. h. sobald $\rho>n$ wird, giebt es keine Substitution unter den $x$, welche $\rho-2$ Werte der $\rho$-wertigen Function $\varphi$ gleichzeitig ungeändert liessen.

Es gilt ferner die bemerkenswerte Erweiterung dieses Satzes: sobald $\rho>n$ ist, enthält $\mathbf{\Sigma}$ keine cyklischen Substitutionen, deren Ordnung eine Prim$z a h l$ ist. Dies beweisen wir folgendermassen: Wenn $\Sigma$ eine Substitution $\tau$ der $\varphi$ einer Primzahlordnung enthielte, und wenn unter $t$ die entsprechende Sub- 
stitution unter den $x_{1}, x_{2}, \ldots, x_{n}$ verstanden wird, dann muss $t$ mindestens einen Cyklus besitzen, welcher dieselbe Ordnung hat, wie $\tau$. Diese Ordnung sei $q$; ferner sei $t=\left(x_{1} x_{2} \ldots x_{q}\right)\left(x_{q+1} \ldots\right) \ldots$ In $\mathbb{S}$ giebt es die Substitution $t_{1}=\left(x_{1} x_{3} x_{2} \ldots x_{q}\right)\left(x_{q+1} \ldots\right) \ldots$, welche aus $t$ durch Transformation mit $\left(x_{2} x_{3}\right)$ hervorgegangen ist. Ihr entspreche $\tau_{3}$ in $\Sigma$. Da nun $t_{1} \cdot t^{-1}=\left(x_{1} x_{2} x_{3}\right)$ wird, so muss $\tau_{1} \cdot \tau^{-1}$ eine Substitution dritter Ord. nung sein, woraus folgt, dass $\tau, \tau_{1}$ gemeinsame Elemente haben, da sonst $\tau_{1} \cdot \tau^{-1}$ von der Ordnung $q$ bleiben würde. Transformirt $\operatorname{man} t_{1} t^{-1}=\left(x_{1} x_{2} x_{3}\right)$ durch $t$ und seine Potenzen, so erkennt man, dass die erhaltenen Substitutionen eine transitive Gruppe entstehen lassen, welche $\left(x_{1} x_{2} x_{3}\right)$ umfasst und folglich die alternirende Gruppe $\mathfrak{F}_{1}$ der verbundenen $q$ Elemente $x_{1}, x_{2}, \ldots x_{q}$ wird. Die entsprechende Gruppe $A_{1}$ in $\Sigma$ enthält höchstens $2 q-1$ Elemente. Sind die Elemente transitiv mit einander verbunden, dann folgt aus Hülfss. VI, dass es nur $q$ sein können und daraus, dass auch die Gruppe $A_{1}$ alternirend in $q$ Elementen $\varphi$ ist und demnach ein $\left(\varphi_{\alpha} \varphi_{\beta} \varphi_{\gamma}\right)$ enthalt. Die transitive Gruppe $\Sigma$ umfasst $\left(\varphi_{\alpha} \varphi_{\beta} \varphi_{\gamma}\right)$, enthält also die alternirende Gruppe der $\rho$ Elemente $\varphi$ und da endlich $\Sigma$ wie $\Im$ zusammengesetzt dagegen $A$ wie $\mathfrak{A}$ einfach ist, so wird $\Sigma$ symmetrisch, $\rho$ ! wird $=n$ ! und $\rho=n$. Intransitiv kann $A_{1}$ nicht sein, da jeder intransitive Teil mindestens $q$ Elemente enthalten müsste.

Als Zusatze ergeben sich: Sobald $\rho>n$ ist, enthäl $\Sigma$ keine Substitution, welche potenzirt eine cyklische Substitution liefert, deren Ordnung gleich einer Primzahl wäre; ebensowenig eine Substitution von einem der Grade 5 oder $\%$.

Die Schlüsse, welche man aus diesen verschiedenen Sätzen auf die Divisorensysteme von Discriminantēn machen kann, sind so ersichtlich, dass sie nicht aufgeführt zu werden brauchen. Nur der erste und einfachste sei hervorgehoben: Wendet man auf die $\rho$ Werte $\varphi_{1}, \varphi_{2}, \ldots, \varphi_{p}$ von $\varphi\left(x_{1}, x_{2}, \ldots, x_{n}\right)$ alle Substitutionen der $x$ an, so bestimmt die hierdurch gebildete zur symmetrischen Gruppe der $x$ isomorfe Gruppe der $\varphi$ eine Classe von Gleichungen $\rho^{\text {ten }}$ Grades; für keine Gattung ihrer Wurzeln besitzt die zugehörige Discriminante Divisorensysteme erster oder zweiter Stufe.

Berlin d. 19 Januar 1883. 